\title{
VARIETIES GENERATED BY MODULAR LATTICES OF WIDTH FOUR
}

\author{
BY RALPH FREESE
}

Communicated by R. S. Pierce, October 28, 1971

The purpose of this paper is to announce some results of the author's thesis on modular width four lattices and their consequences particularly in the field of lattice varieties. Detailed proofs will appear elsewhere.

A variety (equational class) of lattices is said to be finitely based if it is defined by a finite set of identities (see [2] for definitions). Let $M_{n}^{m}$ be the variety generated by all modular lattices of width not exceeding $n$ and length not exceeding $m$, where $m$ and $n$ are cardinals. It is easy to see that if $n_{1}$ and $n_{2}$ are infinite cardinals and $m$ is any cardinal then $M_{m}^{n_{1}}=M_{m}^{n_{2}}$ and $M_{n_{1}}^{m}=M_{n_{2}}^{m}$. Consequently the symbol $\infty$ will be used in place of any infinite cardinal. R. Wille [8] asks for which $m, n$ is $M_{n}^{m}$ finitely based. If $m$
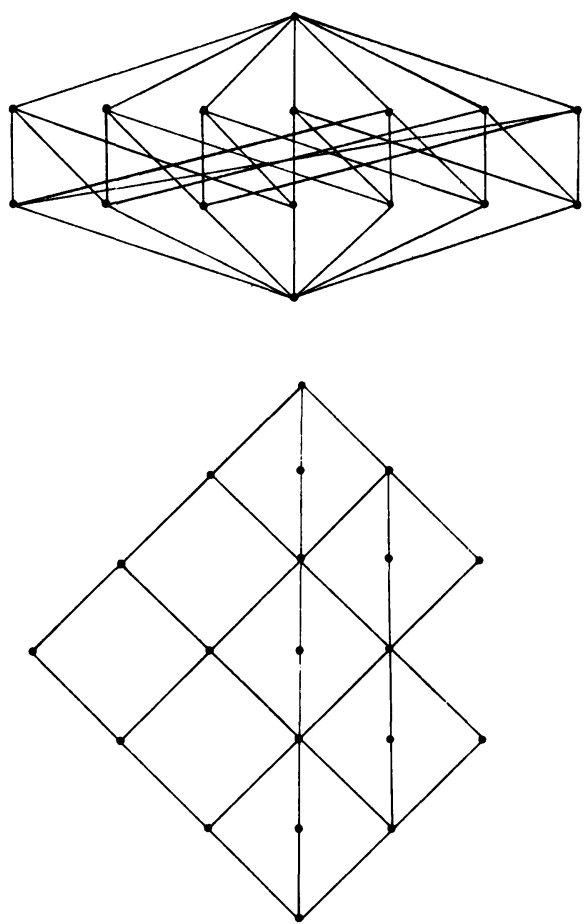

FiguRE 1

AMS 1970 subject classifications. Primary 06A30, 08A15, 06A20; Secondary 08A30. Key words and phrases. Variety (equational class) of lattices, identity, finitely based variety. 

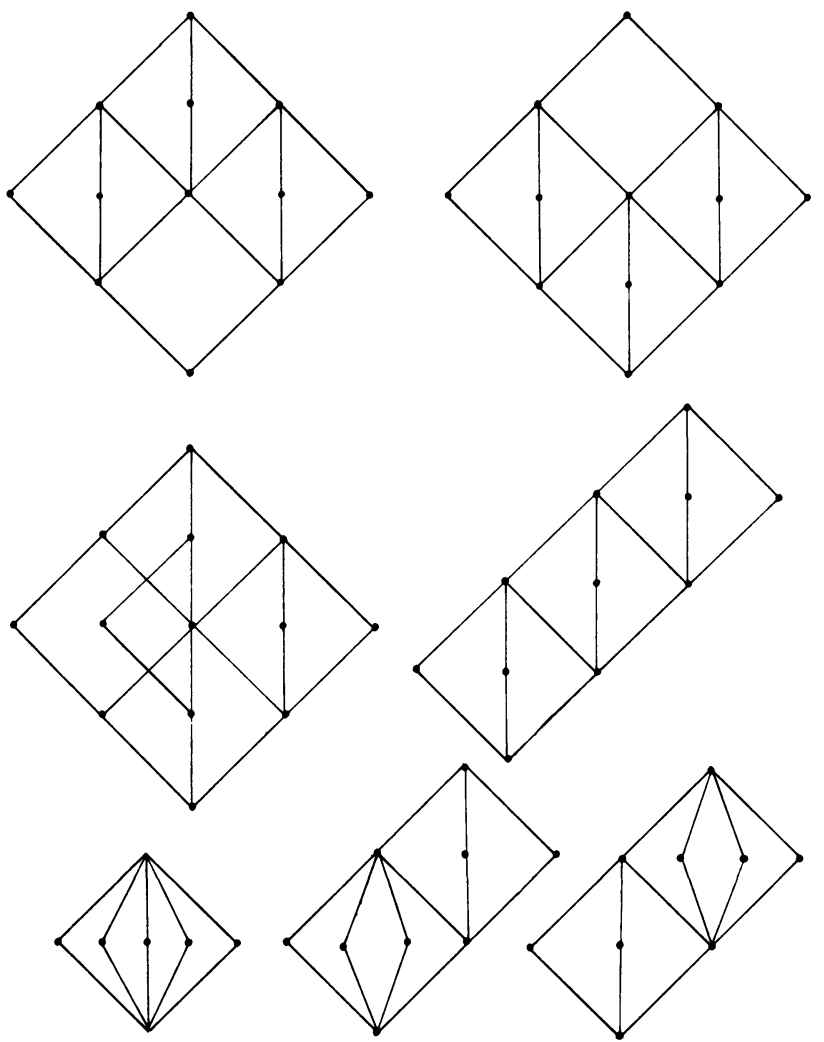

Figure 1 (cont.)

and $n$ are both finite then McKenzie's theorem [7] implies that $M_{n}^{m}$ is finitely based. K. Baker has shown that $M_{\infty}^{m}$ is finitely based for all $m$ [1] and that $M_{n}^{\infty}$ is not finitely based for $5 \leqq n<\infty[1] . M_{1}^{\infty}=M_{2}^{\infty}$ is the class of all distributive lattices and thus finitely based. B. Jónsson [6] has shown that $M_{3}^{\infty}$ is finitely based, leaving only the case of $M_{4}^{\infty}$ unsolved. The main result of this paper is that $M_{4}^{\infty}$ is finitely based. This is an easy corollary of Jónsson's theorem [5] and the following theorem.

THEOREM 1. Let $L$ be a subdirectly irreducible modular lattice of width greater than four. Then one of the nine lattices of Figure 1 is a homomorphic image of a sublattice of $L$.

The techniques used to prove Theorem 1 can also be used to characterize subdirectly irreducible modular lattices of width four.

THEOREM 2. If $L$ is a subdirectly irreducible modular width four lattice then either 


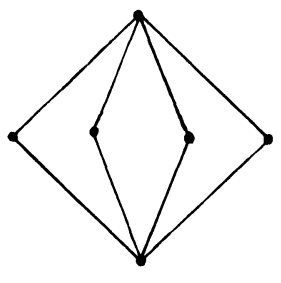

A

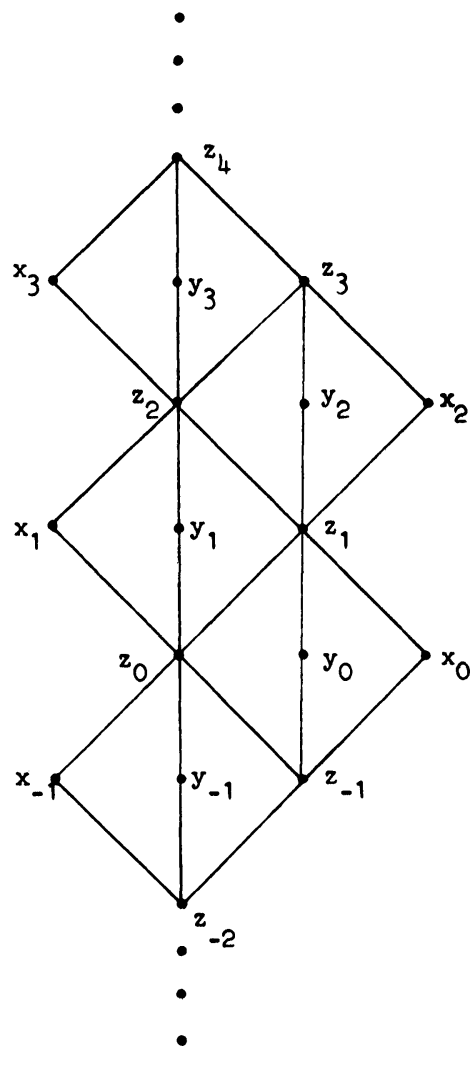

B

FIGURE 2

(i) $L=A$.

(ii) $L$ has a sublattice isomorphic to the quotient sublattice of $B$ lying between $z_{0}$ and $z_{k}$ for some $k$, such that the only other elements that $L$ might have are elements $w_{i}, i=2, \ldots, k-2$, such that $w_{i} \vee z_{i} \in\left\{x_{i+1}, y_{i+1}\right\}$ and $w_{i} \wedge z_{i}=\left\{x_{i-1}, y_{i-1}\right\}$. There is at most one such element for each $i=2, \ldots, k-2$.

(iii) L has a sublattice isomorphic to the sublattice of $B$ consisting of the elements greater than or equal to $z_{0}$ such that the only other elements $L$ can possibly have are the elements $w_{i}, i=2,3, \ldots$, described in (ii) and possibly a greatest element.

(iv) $L$ is the dual to one of the lattices described in (iii).

(v) L has a sublattice isomorphic to $B$ and the only other possible elements are a top element, a bottom element, and the elements $w_{i}, i=0, \pm 1, \pm 2, \ldots$. 
Conversely all the lattices described in (i)-(v) are subdirectly irreducible modular lattices of width four.

Let $F L\left(M_{4}^{\infty}, n\right)$ be the free $M_{4}^{\infty}$ lattice with $n$ generators.

THEOREM 3. If $n$ is finite then $F L\left(M_{4}^{\infty}, n\right)$ is finite. ${ }^{1}$

This theorem is a corollary of Theorem 2 and the following lemma, which is a theorem of universal algebra.

LEMMA. Let $\left(L_{i} \mid i \in I\right)$ be a family of finite lattices such that there are finitely many distinct isomorphic types of lattices. Then if $L$ is a finitely generated sublattice of the direct product of the $L_{i}, i \in I, L$ is finite.

A further consequence of Theorem 2 is the following theorem.

THEOREM 4. There are $2^{\aleph_{0}}$ subvarieties of $M_{4}$.

Notice this has as a corollary that there exists a subvariety of $M_{4}^{\propto}$ which is not finitely based.

\section{REFERENCES}

1. K. Baker, Equational axioms for classes of lattices, Bull. Amer. Math. Soc. 77 (1971), 97-102.

2. G. Birkhoff, Lattice theory, 3rd ed., Amer. Math. Soc. Colloq. Publ., vol. 25, Amer. Math. Soc., Providence, R.I., 1967. MR 37 \# 2638.

3. R. S. Freese, Subdirectly irreducible modular lattices of width four, Notices Amer. Math. Soc. 17 (1970), 1041. Abstract \# 680-A1.

4. Institute of Technology, 1972.

5. B. Jónsson, Algebras whose congruence lattices are distributive, Math. Scand. 21 (1967), 110-121. MR 38 \#5689.

6. Equational classes of lattices, Math. Scand. 22 (1968), 187-196. MR 40 \#66.

7. R. McKenzie, Equational bases for lattice theories, Math. Scand. 27 (1970), 24-38.

8. R. Wille, Primitive Länge und primitive Weite bei modularen Verbänden, Math. Z. 108 (1969), 129-136. MR 39 \#2672.

Department of Mathematics, California institute of Technology, Pasadena, CALIFORNIA 91109

\footnotetext{
${ }^{1}$ Compare with Birkhoff's Problem 46 [2].
} 